\title{
Nun wird wieder selbst gerührt...
}

\author{
Für eine wissenschaftlich korrekte Diagnostik auf \\ Glukosestoffwechselstörungen hat sich eine verträg- \\ liche und standardisierte Fertiglösung für den oralen \\ Glukosetoleranztest in der Praxis bewährt. Diese mit \\ etwa 5 Euro relativ günstige Lösung wird nun seit 1. \\ Januar 2016 von den gesetzlichen Krankenkassen \\ nicht mehr erstattet. Aus verschiedenen Gründen für \\ viele Kollegen ein Unding. Was denken Sie?
}

Inzwischen ist es bereits Gewohnheit - regelmäßig zum Jahreswechsel gibt es zahlreiche Neuerungen für uns Ärzte - und diese betreffen die unterschiedlichsten Bereiche. Zwar wird uns immer wieder versprochen, dass ,innovative und praktikablere Ansätze" für Diagnostik und Therapie sowie Erleichterungen auf bürokratischer Ebene in Sicht sind - in der Realität ist aber genau das Gegenteil der Fall. Ein schönes, aber sehr trauriges, Beispiel dafür ist eine neue Regelung im Bereich „,Sprechstundenbedarf“. Sie betrifft den oralen Glukosetoleranztest (oGTT). Er wird in fast allen hausärztlichen Praxen täglich mit einer vorgefertigten Lösung gemacht, auch in Diabeteszentren und Frauenarztpraxen. Um welche Mengen es sich handelt, ist leider nicht bekannt. Auf Anregung der Fachkommission Diabetes in Bayern (FKDB) werden derzeit aber aktuelle Fallzahlen von der KVB ermittelt.

Nun sollte man meinen, es sei im Interesse aller Beteiligten, dass diese wegweisende Diagnostik auf Glukosestoffwechselstörungen wissenschaftlich korrekt erfolgt. Die Diagnose Diabetes mellitus hat weitreichende Folgen - in erster Linie für den Patienten, aber auch für unser Gesundheitssystem. Ärzte, Krankenkassen und Patienten wissen um die Bedeutung dieser Stoffwechselstörung. Es werden jährlich immense Summen ausgegeben, um die Wirksamkeit und Wirtschaftlichkeit von Diabetesmedikamenten (orale Antidiabetika, Insuline) in großen Studien zu prüfen - welche Kosten hier entstehen ist bekannt! Bei der OGTTDiagnostik handelt es sich um eine Fertiglösung, die zum Preis von etwa 5 Euro bisher über Sprechstundenbedarf bedarf zu beziehen war. Dies ist nun als unwirtschaftlich deklariert, wer die Lösung weiter so bezieht, muss mit Regress rechnen (der meist erst nach 2 Jahren auf den Schreibtisch kommt).

\section{Selbstangerührtes oft schlecht verträglich}

Wer hat diesen Unsinn verhandelt? Wer kommt nur auf so eine Idee? Krankenkassen werben für die Teilnahme der Patienten am Disease Management Programm (DMP) Diabetes Typ 2 die hierfür nötige saubere Diagnostik wollen sie nun aber nicht mehr zahlen. Wie kann das sein? Und vor allem - wie sieht nun die Alternative aus?

Antwort: Wir rühren wieder, so wie vor 20 Jahren, unsere Lösungen selbst an - welch ein „Fortschritt“! Die älteren Kollegen haben es noch gut in Erinnerung, dass insbesondere schwangere Patientinnen darauf häufig erbrechen mussten oder die Glukoselösung erst gar nicht zu Ende trinken konnten. Damit ist die genaue Diagnostik einer Glukosestoffwechselstörung nicht mehr möglich - der Test muss wiederholt werden!

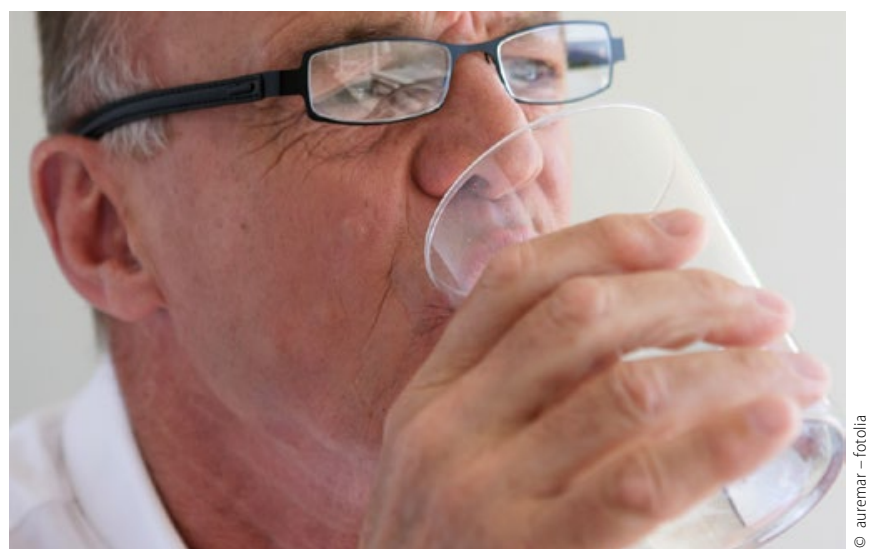

Einen oralen Glukosetoleranztest zu machen, bedeutet eben nicht nur, einfach ein Glas Zuckerwasser zu trinken.

\section{Leider nur eine weitere Fehlerquelle}

Ein weiterer Missstand ist, das die OGTT-Diagnostik oft falsch z.B. mit einem Hand-Blutzucker-Messgerät - erfolgt. Leider stört dies bisher weder Ärzte noch Krankenkassen. So werden Patienten falsch positiv oder falsch negativ diagnostiziert. In einem bundesweiten Survey, der 2009 von Priv. Doz. Dr. Martin Füchtenbusch aus München bei 25\% aller niedergelassener Gynäkologen gemacht wurde, war das erschreckende Ergebnis: In etwa 50\% der Praxen wird beim OGTT die Glukose mit Handmessgeräten bestimmt - diese sind für diagnostische Zwecke aber viel zu ungenau! Täglich sehe ich in meinem Diabeteszentrum Patienten, die mit einer derart falschen Diagnostik überwiesen wurden.

\section{Saubere Diagnostik liegt im Interesse aller}

Wir müssen uns von Seiten der wissenschaftlichen Gremien und als Ärzte aufs Heftigste gegen derartig unsinnige Neuerungen wehren! Eine saubere Diagnostik liegt im Sinne aller Beteiligten. Es kann nicht sein, dass dafür 5 Euro zu viel (,unwirtschaftlich“) sind! Hier wird wieder an der falschen Stelle gespart. Prävention stellen wir uns so nicht vor. Aber vielleicht besteht ja gar kein Interesse daran? Ist diese Neuregelung nur ein „bayrisches Problem"? Wehrt sich hier nur keiner? Warum ist die Fertiglösung „Sprechstundenbedarf“? Sie ist doch ein Diagnostikum so wie z.B. ein Kontrastmittel in der Radiologie! Ich hoffe auf viele $\mathrm{Zu}-$ schriften und Anregungen an mich, nicht nur aus Bayern.

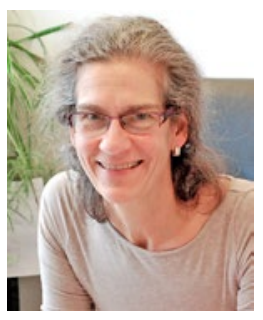

Dr. med. Veronika Hollenrieder

FÄ für Innere Medizin, Diabetologin DDG Vorstandsmitglied der Fachkommission Diabetes Bayern (FKDB)

Hauptstr. 14, 82008 Unterhaching

veronika@praxis-hollenrieder.de 